\title{
FAMILIA Y ESTADO EN SUECIA
}

\author{
Rita Liljestrom \\ (Universidad de Göteberg)
}

Este artículo es la transcripción de una conferencia dictada en la Universidad Autónoma de Barcelona el 19 de mayo de 1981. Problemas en la traducción obligaron a revisar y corregir el primer trabajo, ampliándolo con dos textos de la autora, Roles in Transition, LiberForlag, Suecia, 1978, y A Study of Abortion in Sweden, Royal Ministry for Foreing Affairs, Suecia, 1974.

La revisión y la redacción final del artículo fueron realizados por Judith Astelarra. 
Estoy muy contenta de estar aquí porque viajar por el mundo y encontrarme con otras mujeres es algo que me alegra y me inspira. Comenzaré esta conferencia haciendo un análisis global de la situación en Suecia a partir del siglo pasado. Luego, a partir de estas líneas generales podremos abrir un debate y formular preguntas.

La industrialización se produjo en Suecia ya avanzado el 1800, por lo que el debate sobre la cuestión de la mujer comenzó tardíamente. Una de las razones principales que llevó a plantear la problemática de la mujer era el hecho de que muchas mujeres de la clase alta no estaban casadas y tenían problemas pues necesitaban encontrar trabajo. Alrededor del $40 \%$ de las mujeres de la nobleza sueca eran solteras; esta cifra aumentaba cuanto más alto era el nivel social porque las mujeres de las clases altas tenían más dificultades para encontrar marido. Mientras que los hombres de las clases altas comenzaban ya a casarse con mujeres de las clases más bajas, las mujeres no podían hacer lo mismo.

Esto llevó a que a mediados de 1800 se produjeran una serie de reformas legales que permitieron que estas mujeres se incorporaran a algunos sectores sociales. Se estableció el igual derecho de herencia, libertad de comercio para las mujeres, reformas educacionales, apertura a nuevas profesiones y mayoría de edad para las mujeres solteras, lo que hizo que gran cantidad de mujeres trabajaran en periódicos o se dedicaran a escribir; fue el origen de las primeras feministas suecas.

Cabría preguntarse por qué les concedieron estos derechos a las mujeres en aquella época. Ello se debió a razones ideológicas, pero también a necesidades económicas. En el terreno ideológico, ésta fue una época de ideas liberales que sirvieron de marco a las demandas de transformaciones en la situación de la mujer. Sin embargo, cabe decir también que surgía al mismo tiempo el ideal de la mujer burguesa, es decir, el de la madre y esposa. Aparecen así dos posiciones con respecto a la imagen de la mujer. En el terreno económico se hizo necesario la movilidad del capital. Así, esta necesidad explica la legislación con igual derecho de la herencia: la heren- 
cia sólo por la vía masculina no permitía la suficiente movilización del capital. Asimismo, los hombres de las clases altas no estaban dispuestos a mantener la creciente cantidad de mujeres solteras, por lo que fue necesario abrirles las puertas a las profesiones y a los ingresos.

Aparte de las clases altas había otros dos sectores sociales de mujeres: las agricultoras y las mujeres de clase baja. Con respecto a las agricultoras hubo pocas transformaciones en su vida y en su situación social. La familia rural se mantuvo estable en su organización. En cambio, las mujeres de la clase trabajadora también tuvieron que enfrentarse a innumerables problemas. La transformación de la situación de la mujer se produjo en los dos extremos de las clases sociales: en la mujer de la nobleza y en la de la clase baja.

La desintegración de las familias de la clase baja expuso a sus mujeres a innumerables problemas de índole sexual. La moral tradicional se resquebrajaba, aumentando la cantidad de madres solteras y de abortos. Los índices de mortalidad entre los niños nacidos fuera del matrimonio eran muy altos. Una gran cantidad de mujeres moría de abortos mal hechos, muertes que aparecían formalmente como suicidios. A principios de 1900 , muchos médicos ya estaban preocupados por la magnitud del problema y se llevan a cabo los primeros intentos de modificar la ley de abortos.

En las primeras décadas del siglo $\mathrm{xx}$ comienzan los debates sobre una política sexual que incluyen temas como el aborto y los anticonceptivos. Sin embargo, son los años en que se produce la crisis de población que alcanza su apogeo en la década de los treinta. Esto lleva a que se legisle sobre la familia y a que a partir de 1910 se prohíba el uso de los anticonceptivos. Se produce un compromiso político entre los partidos burgueses que querían aumentar la familia y los socialistas que querían una política de bienestar.

Sin embargo, el debate sobre la sexualidad continúa. En 1938 se levanta la prohibición del uso de anticonceptivos y se continúa con el debate sobre el aborto hasta 1950 , en que se liberaliza la ley. Durante este período aparecen numerosas investigaciones de médicos, asistentes sociales y psiquiatras sobre la problemática de la natalidad. Coincidiendo con la política pronatalista, estos profesionales intentan convencer a las mujeres para que tengan hijos aunque no los deseen. Sin embargo, desde 1938, las mujeres en Suecia se organizaron y lucharon sistemáticamente para que el Estado diera información sobre anticoncepción y sexualidad.

Las exigencias de que la información sexual se haga extensiva a todos los suecos se relacionan con los argumentos en favor del aborto. Se señala el derecho de todos los individuos a nacer de modo que sean bienvenidos. 
Se critica el que las mujeres sean obligadas a ser madres aun sin desearlo, lo que destroza su situación personal. Se analiza el fracaso de los matrimonios hechos por conveniencia debido al embarazo de la mujer. Con estos argumentos se presiona por una política sexual y por una ley sobre el aborto.

Finalmente, en 1975, se promulga una ley que da derecho al aborto totalmente libre en Suecia. Con esto se acaba la primera fase de la política sexual sueca.

En la primera mitad del siglo $\mathrm{xx}$ se producen también transformaciones en la familia. Durante el período de industrialización entre 1863 y 1912, el $20 \%$ de los trabajadores de la industria eran mujeres. Desde el comienzo este mercado de trabajo fue un mercado segregado, ya que las mujeres participaban en tareas que eran una prolongación del trabajo en casa. La mitad de las mujeres trabajadoras trabajaban en la industria textil, y el resto en trabajos auxiliares. El camino de la mujer hacia el mercado del trabajo comenzaba como empleada en una familia agrícola, luego se convertía en una empleada doméstica, luego en ama de casa y, finalmente, como auxiliar de algún servicio.

Pasada la fase de la industrialización las mujeres abandonan el mercado de trabajo: el $90 \%$ de las mujeres suecas se convierten en amas de casa. Esto afecta a todas las clases sociales. Las mujeres de los trabajadores, al poseer un nivel de ingresos suficiente, no trabajan ni en las industrias ni en el servicio doméstico. Las mujeres de clase media, a partir de los años cuarenta ya no cuentan con sirvientes y han de desempeñar ellas mismas las tareas domésticas. Esto las convierte a todas en amas de casa.

En 1920 , se promulga una ley sobre el matrimonio que introduce el matrimonio democrático en Suecia. La mujer se convierte en mayor de edad a los 21 años, tiene derecho a hacer operaciones comerciales sin el permiso del marido y obtiene también la patria potestad sobre los hijos. La mujer casada también se convierte en mayor de edad con todos los derechos que ello comporta. Esta ley es un mecanismo más que impulsa a las mujeres, especialmente las de la clase trabajadora, a regresar al hogar.

Este matrimonio moderno produce dos tipos de individuos, los hombres y las mujeres. En este esquema se puede ver esta separación.
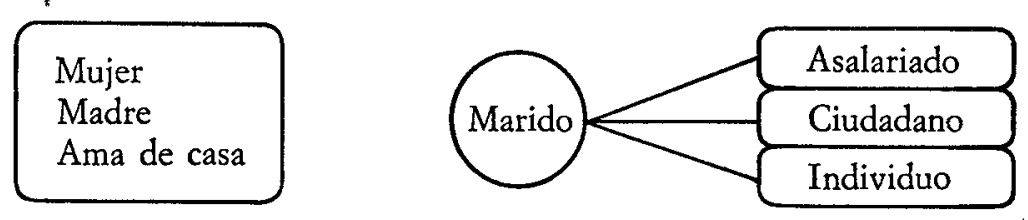
La mujer mantiene un papel unificado de esposa, madre y ama de casa: este papel se refiere a su situación en el hogar. El marido, en cambio, diversifica los roles en que participa en la sociedad convirtiéndose en un asalariado, en un ciudadano y en un individuo. En este sentido, el marido se convierte en el primer ser humano moderno, segregado, diversificado en su personalidad, mientras que la mujer permanece en el papel tradicional que ha tenido siempre.

Después de la primera guerra mundial se produce una situación diferente con un nuevo modelo demográfico y económico. Se consolida la familia del tipo señalado anteriormente, la mayoría de las mujeres se casa, se convierten en amas de casa y tienen niños a una edad muy temprana. $\mathrm{Ha}$ desaparecido el servicio doméstico y esta situación de ama de casa alcanza a la mayoría de las mujeres suecas. Al mismo tiempo se produce una falta de mano de obra en el mercado de trabajo.

Es la falta de mano de obra la que va a conducir al debate sobre la incorporación de la mujer en el trabajo productivo y a la igualdad entre hombres y mujeres. En un primer momento se intenta cubrir la falta de mano de obra mediante la inmigración. Llegan así trabajadores griegos, turcos y yugoslavos. Pero esta mano de obra inmigrante produce gran cantidad de problemas sociales: la integración de los extranjeros en la sociedad sueca no es tan fácil como se había creído.

Cuando se comprendió lo difícil que era la problemática de la fuerza del trabajo mediante el uso de mano de obra inmigrante, se abre un debate en profundidad para la integración de la mujer en el mercado de trabajo. Esto sucede en los años sesenta. Durante toda esta década se discute sobre los factores que impiden a las mujeres incorporarse a la fuerza del trabajo y alcanzar la plenitud de igualdad con los hombres en la sociedad.

Esta discusión produce en los años setenta una nueva legislación que permite a la mujer incorporarse a las tareas sociales. En 1970 se promulga una ley mediante la cual los impuestos pasan a ser individuales en lugar de familiares. En 1972 se crea la Delegación por la Igualdad de la Mujer, cuvo objetivo es generar una política nueva para la mujer tanto en el mercado de trabajo como en otras áreas. En 1974 se promulga una nueva ley del matrimonio y el seguro de los padres. En 1970 se habían ampliado los derechos de herencia a los niños nacidos fuera del matrimonio.

El pago de impuestos individuales es un factor positivo para los matrimonios en que los dos trabajan y se convierte en el factor que induce a las mujeres a entrar en el mercado de trabajo. La Delegación por la Igualdad de las Mujeres emprende varios proyectos cuya finalidad es posibilitar que la mujer se integre en la sociedad. Hay proyectos pilotos para formar e integrar a las mujeres en las profesiones tradicionalmente consideradas 
masculinas. Se hacen estudios para detectar los factores que impiden esta integración y resolverlos. El seguro de padres posibilitaba que el padre pudiera quedarse en casa y mantener el sueldo entero: con ello se pretendía romper con la concepción de que era sólo la madre la responsable de los hijos. Padre y madre podían así compatibilizar el cuidado de los niños con el trabajo remunerado. La nueva ley de matrimonio posibilita el divorcio para el que basta el simple consentimiento de los cónyuges si no hay hijos pequeños; si los hay, el plazo es sólo de 6 meses.

Se llega así a un modelo diferente de la familia democrática: la familia nueva.

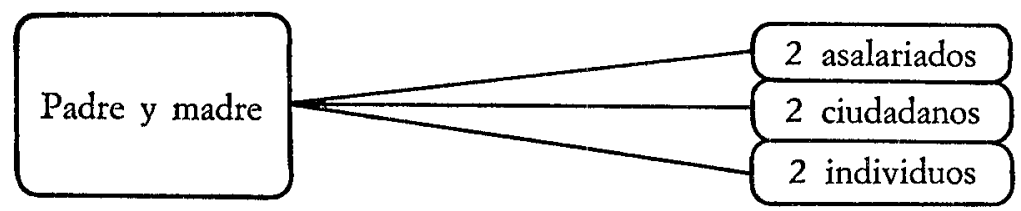

Ahora el ideal de la familia es que ambos cónyuges sean iguales y lo compartan todo. Tenemos, entonces, en la familia, dos padres, que son dos asalariados, dos ciudadanos, y dos individuos. Nos encontramos así ante una familia igualitaria.

Se puede decir, entonces, que la igualdad en Suecia comienza desde arriba, desde el Estado. Que el Estado implementa una legislación y una política que tiende a conseguir la igualdad entre hombres y mujeres. Propone al mismo tiempo un nuevo modelo de familia. Es interesante notar que esta política intenta al mismo tiempo salvaguardar la familia. Se producen así dos vertientes que a veces chocan entre sí: por un lado se considera que lo que hacen el hombre y la mujer es cosa sólo de ellos; por otro lado, se tiende a proteger la familia.

Veamos, a continuación, en qué medida estos cambios producidos por el Estado han producido, en realidad, transformaciones en la sociedad sueca. Nos encontramos, en general, con que la convivencia ha cambiado.

A principios de este siglo la madre soltera estaba muy mal vista en Suecia. Sin embargo, a mediados de los sesenta este patrón había cambiado totalmente. El $90 \%$ de los matrimonios suecos han vivido juntos antes de casarse. En 1978, el 38 \% de los niños nacieron de padres que no estaban casados. Ciertos factores de la sociedad sueca influyen en estos hechos.

En primer lugar existía la tradición de la sociedad agrícola: que los novios pasaran juntos los fines de semana. Aunque se sancionaba a la mujer que quedaba embarazada, no se prohibían las relaciones sexuales en sí. 
En segundo lugar, durante los años sesenta se ha extendido el uso de anticonceptivos al mismo tiempo que la liberalización del aborto permite que las mujeres tengan acceso a él. Finalmente, antes de los años sesenta las empresas solían despedir a las mujeres que se casaban, a pesar de que una ley del año 1939 lo prohibía. Esto hizo que muchas mujeres prefirieran vivir junto a su pareja, sin casarse, para no perder el puesto de trabajo.

Los divorcios también han aumentado en la sociedad sueca. Hasta los años sesenta, el $85 \%$ de los niños habían crecido con sus dos padres. No se sabe aún estadísticamente cuál es la situación, pero sin embargo se puede señalar que antes de los años sesenta existía un divorcio por cada mil habitantes y que actualmente ha subido a trece divorcios.

Se puede decir, entonces, que durante este siglo ha habido cambios muy radicales en Suecia. Cambios en cuanto al mercado de trabajo, en cuanto a la política sexual y en cuanto a la legislación sobre el matrimonio. También han existido transformaciones en las normas de convivencia entre hombres y mujeres en Suecia. Veamos, entonces, cuáles han sido las consecuencias de estas transformaciones.

Analizaremos los problemas que se han producido, en materia de sexualidad, en la familia y en el mercado de trabajo.

En cuanto a la política de sexualidad, se cumplió con una primera fase durante la que se extendió la educación sexual y la información sobre anticoncepción a la mayor parte de la sociedad sueca. Sin embargo, en tiempos recientes numerosos estudios han destacado una gran cantidad de problemas. Todos ellos se refieren a la existencia de un chovinismo masculino que se traduce en violencia y en la opresión sexual de la mujer. Investigaciones recientes han mostrado que el número de violaciones, violencias contra las mujeres y prostitución se han incrementado en Suecia. Esta situación es mucho peor de lo que los suecos habían podido imaginar. $\mathrm{La}$ política de la educación sexual no ha producido una concepción de la sexualidad como una relación humana entre iguales, lo que había sido su meta. La mavoría de los hombres ven a la mujer como a un enemigo, lo que se traduce en violencia sexual. Esto ha llevado a plantearse una segunda fase en la política sobre sexualidad, cuyo énfasis está en ir de la técnica sexual, objetivo de la primera fase, a la calidad de la vida sexual. Es decir, el énfasis ya no se pondrá en el aspecto técnico de la sexualidad, sino en la noción de que la sexualidad es una relación humana entre iguales.

Por lo que respecta a la familia, el modelo ideal de familia nueva propuesto por el Estado no se ha concretado en la realidad. La incorporación de las mujeres a los trabajos productivos remunerados no ha supuesto un cambio en la conducta masculina en el hogar. Los hombres no aportan su cuota de trabajo doméstico ni de cuidado de los niños. El seguro para los 
padres sólo ha sido usado por un $10 \%$ a un $14 \%$ de los nuevos padres. El marido no contribuye en las tareas domésticas del hogar. Así la esposa se ve forzada a realizar el trabajo doméstico y al mismo tiempo a participar en la producción. Esto ha llevado a que la mayoría de las mujeres con niños pequeños sólo trabajen media jornada.

La situación en el mercado de trabajo tampoco ha sido la que se debía esperar como producto de la política que se llevó al respecto. En vez de disminuir, la discriminación y segregación de las mujeres en el mercado del trabajo ha tendido a aumentar. El $66 \%$ de las mujeres trabajan en el área pública, es decir en servicios, mientras que un $65 \%$ de hombres trabajan en el área privada, es decir, en el sector productivo. Se traslada así al mercado laboral la segregación por sexo que existe en la familia. En la actualidad, la elección profesional de los jóvenes no muestra cambios importantes al respecto.

Así, después de 20 años de una política de igualdad promocionada desde arriba, desde el Estado, hoy comenzamos a preguntarnos si el modelo que se impulsó era realmente el adecuado. Las mujeres que hoy están investigando estos problemas en Suecia señalan que esta política de igualdad en realidad se ha traducido en la asimilación de las mujeres a la sociedad masculina, bajo las condiciones que los hombres han impuesto. Esto no sólo ha puesto a las mujeres en una situación de desventaja ante la competencia, sino que también ha tendido a menospreciar lo que eran las tareas y la cultura de la mujer.

El estudio sobre la cultura de la mujer ha llevado a plantearse la problemática de la reproducción humana, del trabajo reproductivo, en un sentido amplio. Algunos estudios históricos contemporáneos intentan reivindicar la historia de las mujeres y relacionarla con la historia oficial. Al mismo tiempo se analizan los dos modelos que los grupos políticos han ofrecido a las mujeres: el modelo liberal y el modelo socialista.

El modelo liberal era un modelo adecuado para las mujeres que poseían educación y podían competir con los hombres bajo sus condiciones. Además, en aquel momento las mujeres de clase alta contaban con la existencia de mujeres del proletariado que servían en sus casas y que les permitían no ocuparse del trabajo doméstico. Así, las mujeres podían gozar de algunas ventajas sin cuestionar el modelo masculino de sociedad.

El modelo socialista, del movimiento obrero, era un modelo que sólo veía las relaciones que había entre el capital y el trabajo y no se ocupaba del trabajo, las relaciones y el mundo de las mujeres. En Suecia los socialdemócratas creían en la productividad, en la tecnología y en un futuro mejor, producto de las transformaciones en el campo de la producción. No se interesaban ni se preocupaban en absoluto por los niños, los viejos, las 
relaciones personales, las formas de vida cotidiana, del consumismo o por la planificación de la vida urbana. Las críticas a la forma de vida, la alternativa de una forma diferente proviene hoy de la clase media bien educada y no de los trabajadores. A comienzos de siglo, los trabajadores habían planteado la existencia de casas colectivas con una cocina comunitaria, con guarderías para cuidar los niños, es decir, un equipamiento urbano y una forma de vivienda que permitieran un estilo de vida diferente. Hoy los trabajadores aspiran a constituir una familia burguesa y tienen posturas muy conservadoras al respecto.

La política de igualdad ha producido, entonces, el aplastamiento de lo que eran los valores del mundo de las mujeres. El cuidado de los niños es el área en que esto aparece más claramente. Actualmente se debate en Suecia la incapacidad de los hombres de dar afecto y cuidar a los niños. El futuro de los niños, la necesidad de entregarles afecto y cuidado, la responsabilidad que tienen, tanto el padre como la madre, de hacerlo, es uno de los debates que tienen lugar en Suecia en la actualidad.

De este modo, el debate actual en Suecia se centra en los problemas que ha producido la política de igualdad que ha desarrollado el Estado, de cara a la familia, la sexualidad y el mercado del trabajo. 